\title{
Approximation of maps into spheres by regulous maps
}

\author{
MaCiej ZielińsKi@
}

\begin{abstract}
Let $X$ be a compact real algebraic set of dimension $n$. We prove that every Euclidean continuous map from $X$ into the unit $n$-sphere can be approximated by a regulous map. This strengthens and generalizes previously known results.
\end{abstract}

Mathematics Subject Classification. 14P05, 14P25.

Keywords. Real algebraic variety, Regulous map, Sphere, Approximation.

1. Introduction. A recent direction of research in real algebraic geometry is to study intermediate classes of maps between continuous and regular maps. Such classes as continuous rational maps, stratified-regular maps, and regulous maps (which often coincide) have been studied in a series of papers $[3,7,8,11-$ 27]. The aim of this note is to strengthen a certain related result of [21] and $[15]$.

We begin by fixing some terminology. A real algebraic variety is a locally ringed space isomorphic to some algebraic subset of $\mathbb{R}^{n}$, for some positive integer $n$, endowed with the Zariski topology and the sheaf of regular functions. It is worth recalling that this class is identical with the class of quasi-projective real varieties, for more detail and information see [4]. A morphism of real algebraic varieties is called a regular map. We will be also interested in the Euclidean topology of such varieties and this is the topology we will mean, unless explicitly stated otherwise, when using topological notions. By a smooth map we understand a map of class $\mathcal{C}^{\infty}$.

Let $X$ be a real algebraic variety. A stratification $\mathcal{S}$ of $X$ is a finite collection of pairwise disjoint Zariski locally closed subvarieties whose union is equal to $X$. A map $f: X \rightarrow Y$ of real algebraic varieties is said to be regulous if it is continuous and if there exists some stratification $\mathcal{S}$ of $X$ such that $\left.f\right|_{S}$ is a regular map for every $S \in \mathcal{S}$. We denote the set of all regulous maps between $X$ and $Y$ by $\mathcal{R}^{0}(X, Y)$. We shall treat $\mathcal{R}^{0}(X, Y)$ as a subspace of the space 
$\mathcal{C}(X, Y)$ of all continuous maps endowed with the compact-open topology. Note that regulous maps in the sense of our definition were called stratifiedregular in [21] and the follow-up papers [20,22]. This definition is different but equivalent to that of [7] where the terminology was introduced, see [21, Remark 2.3] or [24].

Each regulous map is also continuous rational-i.e. $f$ is continuous and $\left.f\right|_{X^{0}}$ is regular for some Zariski open dense subset $X^{0} \subset X$. While the converse is false in general, it is true if $X$ is nonsingular, see [11].

Let us first recall the following result contained in [15].

Theorem 1.1. Let $X$ be a compact nonsingular real algebraic variety of dimension $p \geq 1$. Then the set $\mathcal{R}^{0}\left(X, \mathbb{S}^{p}\right)$ is dense in $\mathcal{C}\left(X, \mathbb{S}^{p}\right)$.

A related weaker result allowing for a singular $X$ is contained in [21]:

Theorem 1.2. Let $X$ be a compact real algebraic variety of dimension $p \geq 1$. Then any continuous map $X \rightarrow \mathbb{S}^{p}$ is homotopic to a regulous map.

Our aim is to strenghten Theorem 1.2 by showing that the nonsingularity assumption of Theorem 1.1 is unnecessary.

Theorem 1.3. Let $X$ be a compact real algebraic variety of dimension $p \geq 1$. Then the set $\mathcal{R}^{0}\left(X, \mathbb{S}^{p}\right)$ is dense in $\mathcal{C}\left(X, \mathbb{S}^{p}\right)$.

It is well known that analogous results do not hold if regulous maps in Theorems 1.1, 1.2, and 1.3 are replaced with regular maps. For example, a continuous map $\mathbb{S}^{1} \times \mathbb{S}^{1} \rightarrow \mathbb{S}^{2}$ is homotopic to a regular map if and only if it is null-homotopic, cf. [5].

2. Proof of the main theorem. We shall use the concept of the algebraic cohomology classes of a real algebraic variety which we recall now. Let $X$ be a compact nonsingular real algebraic variety. A class in $H^{*}(X ; \mathbb{Z} / 2)$ is said to be algebraic if it is Poincaré dual to a homology class in $H_{*}(X, \mathbb{Z} / 2)$ represented by an algebraic subset. The set $H_{\text {alg }}^{*}(X ; \mathbb{Z} / 2)$ of all algebraic cohomology classes is a subring of $H^{*}(X ; \mathbb{Z} / 2)$ and if $f: X \rightarrow Y$ is a regular map, then the induced map $f^{*}$ in cohomology maps $H_{\text {alg }}^{*}(Y ; \mathbb{Z} / 2)$ into $H_{\text {alg }}^{*}(X, \mathbb{Z} / 2)$, cf. $[2,4,6]$.

An important tool we need is [15, Lemma 2.2], which allows controlled approximation of continuous maps into projective space by regular maps. We restate it here for convenience.

Lemma 2.1. Let $X$ be a compact nonsingular real algebraic variety, and let $A$ be a Zariski closed subvariety of $X$. Let $f: X \rightarrow \mathbb{P}^{n}(\mathbb{R})$ be a continuous map whose restriction $\left.f\right|_{A}: A \rightarrow \mathbb{P}^{n}(\mathbb{R})$ is a regular map. Assume that

$$
f^{*}\left(H^{1}\left(\mathbb{P}^{n}(\mathbb{R}) ; \mathbb{Z} / 2\right)\right) \subset H_{\text {alg }}^{1}(X ; \mathbb{Z} / 2) .
$$

Then one can find a regular $g: X \rightarrow \mathbb{P}^{n}(\mathbb{R})$ arbitrarily close to $f$ and satisfying $\left.g\right|_{A}=\left.f\right|_{A}$ (i.e. every neigborhood of $f$ in $\mathcal{C}\left(X, \mathbb{P}^{n}(\mathbb{R})\right.$ ) contains such a map).

We are now ready to prove Theorem 1.3 
Proof. Let $f$ be any map in $\mathcal{C}\left(X, \mathbb{S}^{p}\right)$. Treating $X$ as a closed subset of $\mathbb{R}^{m}$ for some $m \in \mathbb{N}$, one can find a smooth map $f_{0}: U \rightarrow \mathbb{S}^{p}$ defined on some neighborhood $U$ of $X$ in $\mathbb{R}^{m}$ such that $\left.f_{0}\right|_{X}$ is arbitrarily close to $f$. Let $\Sigma$ denote the singular locus of $X$. Then $f_{0}(\Sigma) \subsetneq \mathbb{S}^{p}$, since $\operatorname{dim} \Sigma<p$. This allows us to approximate $\left.f_{0}\right|_{\Sigma}$ by regular maps using the stereographic projection and Weierstrass approximation theorem. We can therefore reduce the proof (by replacing $f$ with suitably modified $f_{0}$ ) to the case where $f$ is a restriction of a smooth map defined on a neighborhood of $X$ in $\mathbb{R}^{m}$ and $\left.f\right|_{\Sigma}$ is regular with $f(\Sigma) \subsetneq \mathbb{S}^{p}$. Then, by Sard's theorem, there exists an $s_{0} \in \mathbb{S}^{p} \backslash f(\Sigma)$ which is a regular value of the smooth map $\left.f\right|_{X \backslash \Sigma}$.

By Hironaka's resolution of singularities theorem $[9,10]$, there exists a finite composition of blowups $\pi: Y \rightarrow X$ over $\Sigma$ with $Y$ nonsingular. The restriction $\bar{\pi}: Y \backslash \pi^{-1}(\Sigma) \rightarrow X \backslash \Sigma$ of $\pi$ is then a biregular isomorphism and $s_{0}$ is a regular value of the smooth map $f \circ \bar{\pi}$. Letting $F=(f \circ \pi)^{-1}\left(s_{0}\right)=(f \circ \bar{\pi})^{-1}\left(s_{0}\right)$, consider the blowup of $Y$ with center $F$, which we will denote $\sigma: B(Y, F) \rightarrow Y$, and the blowup $\tau: B\left(\mathbb{S}^{p}, s_{0}\right) \rightarrow \mathbb{S}^{p}$ of $\mathbb{S}^{p}$ over $s_{0}$. Since $\operatorname{dim} X=p$, the set $F$ is finite as a fiber of the smooth map $f \circ \bar{\pi}$ over its regular value $s_{0}$, hence $B(Y, F)$ is a real algebraic variety. Finiteness of $F$ and the fact that $f \circ \pi$ is also a smooth map allow us to apply [1, Lemma 2.5.9] in order to construct a smooth lifting $g$ of $f \circ \pi$ to a map between $B(Y, F)$ and $B\left(\mathbb{S}^{p}, s_{0}\right)$ making the following diagram commute:

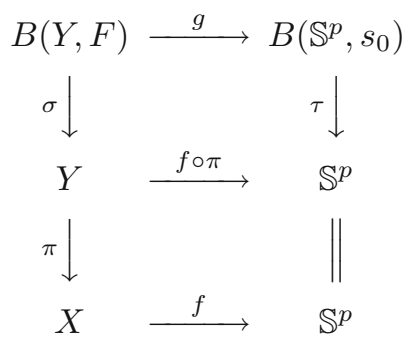

Our aim for now is to find a regular map $H: B(Y, F) \rightarrow B\left(\mathbb{S}^{p}, s_{0}\right)$ arbitrarily close to $g$ in $\mathcal{C}\left(B(Y, F), B\left(\mathbb{S}^{p}, s_{0}\right)\right)$ in such a way that the map $\tilde{f}: X \rightarrow \mathbb{S}^{p}$ making the following diagram commute will be regulous and close to $f$ :

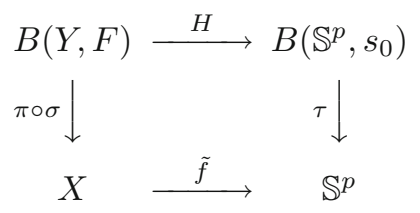

Let $D=\sigma^{-1}(F)$ and $E=\tau^{-1}\left(s_{0}\right)$. Then $D$ and $E$ are nonsingular algebraic hypersurfaces in the respective blowups. Let $u \in H^{1}(B(Y, F) ; \mathbb{Z} / 2)$ be the cohomology class Poincaré dual to the homology class in $H_{*}(B(Y, F) ; \mathbb{Z} / 2)$ represented by $D$ and let $v$ be the class in $H^{1}\left(B\left(\mathbb{S}^{p}, s_{0}\right) ; \mathbb{Z} / 2\right)$ dual to the class in $H_{*}\left(B\left(\mathbb{S}^{p}, s_{0}\right) ; \mathbb{Z} / 2\right)$ represented by $E$. Recall that there exists a biregular isomorphism $\varphi: B\left(\mathbb{S}^{p}, s_{0}\right) \rightarrow \mathbb{P}^{p}(\mathbb{R})$ such that

$$
\varphi(E)=\mathbb{P}^{p-1}(\mathbb{R}) \subset \mathbb{P}^{p}(\mathbb{R}) .
$$


Hence $H^{1}\left(B\left(\mathbb{S}^{p}, s_{0}\right) ; \mathbb{Z} / 2\right)=\varphi^{*}\left(H^{1}\left(\mathbb{P}^{p}(\mathbb{R}) ; \mathbb{Z} / 2\right)\right) \cong \mathbb{Z} / 2$ with $v$ the generator. Since $g$ is transverse to $E$ and $D=g^{-1}(E)$ we have $u=g^{*}(v)$ (cf. [6, Proposition 2.15]) and it follows that

$$
g^{*}\left(H^{1}\left(B\left(\mathbb{S}^{p}, s_{0}\right) ; \mathbb{Z} / 2\right)\right) \subset H_{\mathrm{alg}}^{1}(B(Y ; F) ; \mathbb{Z} / 2) .
$$

Let $i: D \rightarrow B(Y, F)$ and $j: E \rightarrow B\left(\mathbb{S}^{p}, s_{0}\right)$ be the inclusion maps and let $\bar{g}: D \rightarrow E$ be the map induced by the restriction of $g$ to $D$. Then, since $g \circ i=j \circ \bar{g}$ we have $\bar{g}^{*}\left(j^{*}(v)\right)=i^{*}\left(g^{*}(v)\right)=i^{*}(u)$. This allows us to apply Lemma 2.1 to approximate $\bar{g}$ by regular maps. Indeed, since by $(1) H^{1}(E ; \mathbb{Z} / 2)$ is generated by $j^{*}(v)$ and by definition $i^{*}(u)$ is in $H_{\text {alg }}^{1}(D ; \mathbb{Z} / 2)$, we have

$$
\bar{g}^{*}\left(H^{1}(E ; \mathbb{Z} / 2)\right) \subset H_{\text {alg }}^{1}(D ; \mathbb{Z} / 2) .
$$

Therefore, there exists a regular map $r: D \rightarrow E$ arbitrarily close to $\bar{g}$ in $\mathcal{C}(D, E)$.

Let $L=(\pi \circ \sigma)^{-1}(\Sigma)$ and let $h: \Sigma \rightarrow B\left(\mathbb{S}^{p}, s_{0}\right)$ be the regular map given by $\left(\left.\tau\right|_{\tau^{-1}\left(\mathbb{S}^{p} \backslash\left\{s_{0}\right\}\right)}\right)^{-1} \circ\left(\left.f\right|_{\Sigma}\right)$. We now extend $j \circ r$ to a smooth map $G: B(Y, F) \rightarrow$ $B\left(\mathbb{S}^{p}, s_{0}\right)$ close to $g$ such that $\left.G\right|_{L}=\left.(h \circ \pi \circ \sigma)\right|_{L}$ (this is possible since the sets $E$ and $L$ are disjoint). If $G$ is close enough to $g$, we have $g^{*}=G^{*}$ and hence by (2) we can apply Lemma 2.1 to $G$ with $A=D \cup L$. This gives a regular map $H: B(Y, F) \rightarrow B\left(\mathbb{S}^{p}, s_{0}\right)$ which is close to $g$ and satisfies $\left.H\right|_{L}=\left.(h \circ \pi \circ \sigma)\right|_{L}$ and $H(D) \subset E$. The latter shows that the map $\bar{H}: Y \rightarrow \mathbb{S}^{p}$ given by

$$
\bar{H}(y)= \begin{cases}s_{0}, & \text { for } y \in F, \\ \tau\left(H\left(\sigma^{-1}(y)\right)\right), & \text { for } y \notin F,\end{cases}
$$

is continuous and well-defined. Moreover $\bar{H}(y)=f \circ \pi(y)$ for $y \in \pi^{-1}(\Sigma)$. Also note that $\bar{H}$ is close to $f \circ \pi$, since $\left.(\tau \circ H)\right|_{Y \backslash F}$ is close to $\tau \circ f \circ \pi$. Therefore, the map $\tilde{f}: X \rightarrow \mathbb{S}^{p}$ given by

$$
\tilde{f}(x)= \begin{cases}f(x), & \text { for } x \in \Sigma, \\ \bar{H}\left(\pi^{-1}(x)\right), & \text { for } x \notin \Sigma,\end{cases}
$$

is well-defined, continuous, and can be chosen arbitrarily close to $f$.

It remains to check that $\tilde{f}$ is regulous. Since $\left.\tilde{f}\right|_{\Sigma}$ is regular as is $\tilde{f}$ in restriction to the finite set $f^{-1}\left(s_{0}\right)$, it is enough to show $\left.\tilde{f}\right|_{X \backslash A}$ is regular where $A=\Sigma \cup f^{-1}\left(s_{0}\right)$. Since we have $\left.\tilde{f}\right|_{X \backslash A}=\tau \circ H \circ\left(\left.\sigma\right|_{Y \backslash F}\right)^{-1} \circ\left(\left.\pi\right|_{X \backslash \Sigma}\right)^{-1}$ with all the maps on the right-hand side regular, the proof is finished.

Acknowledgements. The author was partially supported by the National Science Centre (Poland) under Grant Number 2014/15/B/ST1/00046.

Open Access. This article is distributed under the terms of the Creative Commons Attribution 4.0 International License (http://creativecommons.org/licenses/ by/4.0/), which permits unrestricted use, distribution, and reproduction in any medium, provided you give appropriate credit to the original author(s) and the source, provide a link to the Creative Commons license, and indicate if changes were made. 


\section{References}

[1] S. Akbulut and H. King, Topology of Real Algebraic Sets, Mathematical Sciences Research Institute Publications, 25, Springer, New York, 1992.

[2] R. Benedetti And A. Tognoli, Remarks and counterexamples in the theory of real algebraic vector bundles and cycles, In: Real Algebraic Geometry and Quadratic Forms (Rennes, 1981), 198-211, Lecture Notes in Math., 959, Springer, Berlin, 1982.

[3] M. Bilski, W. Kucharz, A. Valette, and G. Valette, Vector bundles and regulous maps, Math. Z. 275 (2013), 403-418.

[4] J. Bochnak, M. Coste, And M.-F. Roy, Real Algebraic Geometry, Ergebnisse der Mathematik und ihrer Grenzgebiete (3), 36, Springer, Berlin, 1998.

[5] J. Bochnak and W. Kucharz, Realization of homotopy classes by algebraic mappings, J. Reine Angew. Math. 377 (1987), 159-169.

[6] A. Borel and A. Haefliger, La classe d'homologie fondamentale d'ub espace analytique, Bull. Soc. Math. France 89 (1961), 461-513.

[7] G. Fichou, J. Huisman, F. Mangolte, And J.-Ph. Monnier, Fonctions régulues, J. Reine Angew. Math. 718 (2016), 103-151.

[8] G. Fichou, J.-P. Monnier, and R. Quarez, Continuous functions in the plane regular after one blowing up, Math. Z. 285 (2017), 287-323.

[9] H. Hironaka, Resolution of singularities of an algebraic variety over a field of characteristic zero, Ann. of Math. 79 (1964), 109-326.

[10] J. Kollár, Lectures on Resolution of Singularities, Annals of Mathematics Studies, 166, Princeton University Press, Princeton, NJ, 2007.

[11] J. Kollár AND K. NowaK, Continuous rational functions on real and p-adic varieties, Math. Z. 279 (2015), 85-97.

[12] J. Kollár, W. Kucharz, and K. Kurdyka, Curve-rational functions, to appear in Math. Ann.

[13] W. Kucharz, Rational maps in real algebraic geometry, Adv. Geom. 9 (2009), $517-539$.

[14] W. Kucharz, Regular versus continuous rational maps, Topology Appl. 160 (2013), 1086-1090.

[15] W. Kucharz, Approximation by continuous rational maps into spheres, J. Eur. Math. Soc. 16 (2014), 1555-1569.

[16] W. Kucharz, Continuous rational maps into the unit 2-sphere, Arch. Math. 102 (2014), 257-261.

[17] W. Kucharz, Some conjectures on stratified-algebraic vector bundles, J. Singul. 12 (2015), 92-104.

[18] W. Kucharz, Continuous rational maps into spheres, Math. Z. 283 (2016), $1201-1215$.

[19] W. Kucharz, Stratified-algebraic vector bundles of small rank, Arch. Math. 107 (2016), 239-249. 
[20] W. Kucharz and K. Kurdyka, Some conjectures on continuous rational maps into spheres, Topology Appl. 208 (2016), 17-29.

[21] W. Kucharz And K. Kurdyka, Stratified-algebraic vector bundles, to appear in J. Reine Angew. Math.

[22] W. Kucharz and K. Kurdyka, Comparison of stratified-algebraic and topological K-theory, arXiv:1511.04238 [math.AG].

[23] W. Kucharz And K. KurdyKa, Linear equations on real algebraic surfaces, arXiv:1602.01986 [math.AG].

[24] W. KucharZ And M. Zieliński, Regulous vector bundles, arXiv:1703.05566 [math.AG].

[25] J.-P. Monnier, Semi-algebraic geometry with rational continuous functions, arXiv:1603.04193 [math.AG].

[26] K.J. NowAK, Some results of algebraic geometry over Henselian rank one valued fields, Sel. Math. New Ser. 28 (2017), 455-495.

[27] M. ZiELIŃski, Homotopy properties of some real algebraic maps, Homology Homotopy Appl. 18 (2016), 287-294.

MACIEJ ZielińSKI

Institute of Mathematics, Faculty of Mathematics and Computer Science Jagiellonian University

ul. Łojasiewcza 6

30-348 Kraków

Poland

e-mail: Maciej.Zielinski@im.uj.edu.pl

Received: 26 May 2017 